\title{
MicroED application for GPCRs: structure of the human adenosine A2A receptor determined from a single nanocrystal in LCP \\ Anna Shiriaeva ${ }^{1}$ \\ ${ }^{1}$ No affiliation given \\ shiriaev@g.ucla.edu
}

G-protein coupled receptors (GPCRs) constitute a large superfamily of membrane proteins that are responsible for initiation of signaling cascades inside the cells in response to extracellular cues. GPCRs are highly significant pharmacological targets: over 30\% of all approved therapeutic drugs act via GPCRs. Adenosine A2A receptor is one of GPCRs targeted for the treatment of cardiovascular, neurodegenerative disorders.

Crystallization of GPCRs is usually carried out in the lipidic cubic phase (LCP) in order to provide a stabilizing lipidic environment for the membrane protein crystals. In many cases, GPCRs in LCP form microcrystals that are too small for traditional X-ray crystallography. Such microcrystals can be targeted by microED approach. However, high viscosity of LCP makes traditional microED sample preparation insurmountable. In order to overcome this obstacle, we converted the LCP into the sponge phase by the excess of PEG400, prepared grids and used cryoFIB milling to achieve the optimal sample thickness.

Our approach allowed to determine the structure of adenosine A2A receptor at $2.8 \AA$ resolution. The overall structure of A2AAR is consistent with past reports. We were able to resolve the ligand ZM241385 in the orthosteric pocket and four surrounding cholesterol molecules bound to the receptor on the extracellular side.

MicroED data was collected from a single lamella in the continuous rotation mode over wedges of approximately $70^{\circ}$. Total diffracting volume was less than $1 \mu \mathrm{m} 3(0.2 \times 2 \times 2 \mu \mathrm{m})$, which is lower than any other method used for GPCR structure determination to date. The average exposure was 2 e- $\AA-2(\sim 7.4$ MGy) to minimize radiation damage.

This investigation represents a leap forward in the use of MicroED and FIB milling for GPCR structure determination. Our approach can be extended to structure determination of novel GPCRs and membrane proteins from microcrystals that would otherwise be impossible by other crystallographic approaches. 